\title{
MDR Pseudomonas aeruginosa in Nosocomial Infection: Burden in ICU and Burn Units of a Tertiary Care Hospital
}

\author{
Usha Verma*, Smita Kulshreshtha and P.K. Khatri
}

Department of Microbiology, Dr. S. N. Medical College, Jodhpur, Rajasthan, India

*Corresponding author

\section{Keywords}

Nosocomial, MDR, Resistance

Article Info

Accepted:

12 December 2017

Available Online:

10 January 2018
A B S T R A C T

Pseudomonas aeruginosa is an opportunistic pathogen and is the leading cause of nosocomial infections especially among patients admitted to ICU and burn units. It has natural resistance as well as acquired multi drug resistance to various antibiotics leading to increased morbidity and mortality. Aim of the study is to isolate P. aeruginosa from burn and ICU patients and to evaluate multidrug resistance. Samples were collected from 100 patients from both ICUs and burn unit for a period of 15 months. Samples were processed in the Microbiology laboratory by standard bacteriological methods. Growths of $P$. aeruginosa were tested for antibiotic susceptibility testing as per CLSI guidelines. Out of 100 patients, $P$. aeruginosa were recovered from burn and ICU patients $24 \%$ and $18 \%$ respectively. Resistance rates to various antibiotics in burn unit were as follows: Piperacillin/Tazobactum (33.3\%), Piperacillin (75\%), Aztreonam (75\%), Imipenem (33.3\%), Amikacin (83.3\%), Ciprofloxacin (58.3\%), Colistin (16.7\%), Ceftazidime (75\%), Cefepime (66.7\%). In ICU unit resistance rates were Piperacillin/ Tazobactum (33.3\%), Piperacillin (77.8\%), Aztreonam (66.7\%), Imipenem (22.2\%), Amikacin (55.6\%), Ciprofloxacin (44.4\%), Colistin (11.11\%), Ceftazidime (55.6\%), Cefepime (44.4\%). All $21 \%$ isolates were MDR patients. High rates of MDR in burn and ICU units suggest restrictions of selected antibiotics and reframing the infection control policies. A regular screening of ICU can help to combat the emergence of MDR strains of P. aeruginosa and reducing the spread of drug resistance in pathogens.

\section{Introduction}

Pseudomonas aeruginosa being an opportunistic human pathogen, is the leading cause of nosocomial infections, especially among patients who are admitted to intensive care units (ICU). It has been implicated in diverse nosocomial infections like nosocomial pneumonias, urinary tract infections (UTIs), skin and soft tissue infections, in severe burns and in infections in immunocompromised individuals. In recent years, a considerable increase in the prevalence of multidrug resistance (MDR) in $P$. aeruginosa has been noticed, which is related to high morbidity and mortality (Babay, 2001-2005).

Burn patients are at risk for developing sepsis secondary to pneumonia, catheter-related infections, and suppurative thrombophlebitis. 
(Appelgren et al., 2002) Risk factor for Pseudomonas is extent, severity and location and care of injuries, exposure to invasive procedures. Although present techniques of burn wound care have significantly reduced the incidence of infections, severely burned patients may still develop life-threatening infections (Steinsträsser et al., 2007).

The unique feature of $P$. aeruginosa is its resistance to a variety of antibiotics which is attributed to a low permeability of the cell wall, the production of inducible cephalosporinases, an active efflux and a poor affinity for the target (DNAgyrase). (AlTawfiq, 2007) Multi drug resistance $P$. aeruginosa (MDRPa) is defined as an isolate intermediate or resistant to at least three groups of antibiotics among $\beta$ lactams, carbapenem, and Fluoroquinolones (Marilee and Obritsch, 2005).

The risk for acquiring MDR organisms may be related to the number of carriers in the same ward as well as to individual risk factors, such as in burn patient characteristics like burn extent, severity, location, care of burn injuries exposure to invasive procedures and in ICU patients characteristics like nature and duration of invasive, procedures used, length of ICU, hospital stay, antibiotic treatment and mortality (Carmeli et al., 2002). Resistance to aminoglycosides was not used as these are not used as treatment in the single drug therapy for Pseudomonas.

The main objectives of this study include to isolate Pseudomonas aeruginosa from burn and I.C.U. patients. And to find antibiotic resistance and multidrug resistance among Pseudomonas isolates. Also to detect the prevalence of Pseudomonas aeruginosa.

\section{Materials and Methods}

A total 100 patients were selected.50 each from both ICUs and burn unit. This study was conducted in M. G. Hospital, Microbiology lab, associated with Dr. S. N. Medical College, Jodhpur, Rajasthan, from May 2015 to July 2016 (15 months). Structured questionnaires were used to extract data from the patient's case notes and/or the patient directly; the information included were: patient's name, age, gender, residential status, registration number, antibiotics or drug history, any associated risk factor like diabetes mellitus, AIDS, cancer and immunosuppressive drugs therapy and relatively clinical history taken.

This prospective study was undertaken with 100 samples (pus, urine, sputum, vaginal swab, blood, pleural fluid). 50 samples each from admitted to both ICU and burn units. A total of 21 out of 100 patients (21\%) from whom various clinical samples (such as, urinary, pleural fluid, sputum, surgical site infections, tracheal aspiration samples from ICU and pus swabs from burn unit) were taken. All type of samples was transported to microbiology lab within 1 hour. In case of delay, transported sample were kept in refrigerator at $4^{\circ} \mathrm{C}$ for 24 hours to reduce growth of contamination. Specimen was inoculated on MacConkey agar, Blood agar and Nutrient agar plates and the plates were incubated at $37^{\circ} \mathrm{C}$ over night. The accurate isolate and identification of Pseudomonas aeruginosa according to the standard microbiological techniques done. (Koneman, 2006) Pseudomonas aeruginosa isolates were confirmed by certain biochemical tests including (Growth at $42^{\circ} \mathrm{C}$, Catalase test $+v e$, Oxidase test $+v e$, Indole test -ve, Methyl red test -ve, Citrate utilization test $+v e$, Urease test -ve, Triple sugar iron test $\mathrm{K} / \mathrm{K}$ or $\mathrm{K} /$ no change without $\mathrm{H}_{2} \mathrm{~S}$ gas production, pigment production). In addition to these tests, sugar fermentation tests including Glucose, Sucrose, Maltose were also performed. The $P$. aeruginosa ATCC 27853 strain was used as the quality control. Routine panel of antimicrobial sensitivity tests were performed 
by the Kirby-Bauer disc diffusion method and the results were interpreted according to Clinical and Laboratory Standard Institute 2014 guideline (CLSI) (Clinical and Laboratory Standards Institute, 2014). Commercially available disks from Himedia were used. All media and antibiotic disc were procured from $\mathrm{Hi}$ media Lab Mumbai, antibiotic potency was standardised antigen reference strain $E$. coli (beta lactamase) as positive control, reference strain of Pseudomonas aeruginosa ATTC 27853 as negative control. P. aeruginosa were tested against Piperacillin-Tazobactam 100/10 $\mu$ g, Ceftazidime $30 \mu \mathrm{g}, \quad$ Cefepime $30 \mu \mathrm{g}$, Piperacillin $100 \mu \mathrm{g}$, Colistin $10 \mu \mathrm{g}$,

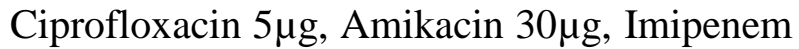

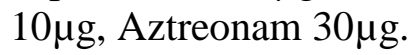

\section{Results and Discussion}

Out of 50 clinical samples processed from the burn patient, $P$. aeruginosa was most common isolated from 12 samples, followed by Staphylococcus aureus., Coagulase negative Staphylococus., Klebsiella sp., Acinetobacter spp., Escherichia coli, Citrobacter sp., Enterobacter sp., Enterococcus faecalis and Proteus sp (Fig. 1).

In I.C.U., out of 50 clinical samples processed, $P$. aeruginosa was isolated from 9 samples.

Antibiotic resistance pattern showed that all 21 isolates were multidrug resistance Pseudomonas aeruginosa (MDRPa).

Infection due to MDRPa has become a challenge in clinical practice and is not uncommon (Naseer et al., 2003). In MDRPa infections, susceptibility testing for antimicrobials that are not tested routinely and antibiotic synergy studies should be considered for synergistic effects.
Furthermore, combination therapy may exert a selection pressure that allows only sub populations with the reduced virulence to be expressed (Steinsträsser et al., 2007).

In burn patients out of 50 samples, the prevalence of $P$. aeruginosa was $24 \%$. A study carried out by Upadhyay et al., in 2014 showed that $17 \%$ of $P$. aeruginosa among infected burns patients, which is almost similar to present study (Upadhaya, 2014).

In ICU patients out of 50 samples, the prevalence of $P$. aeruginosa was $18 \%$. A study carried out by Masomeh Bayani et al., in 2013 and Lutfu savas et al., showed that 60\% and $12.7 \%$ respectively of $P$. aeruginosa among infected ICU patients (Masomeh Bayani et al., 2013; Lutfu savas et al., 2005)

MDRPa have commonly been reported as colonizers of the wounds or the cause of nosocomial outbreaks infection in burn units. Present study shows the highest resistance to Amikacin (83.3\%), Piperacillin (75\%), Ceftazidime (75\%) and Aztreonam (75\%), similar study done by Kamaria et al., showed that Amikacin, Piperacillin, Ceftazidime and Aztreonam were 85.13\%, 72.97\%, 81.08\% and $83.78 \%$ resistant respectively (Kamaria et al., 2016) $P$. aeruginosa shows intermediate resistance to Ciprofloxacin $(58.3 \%)$ and Cefepime (66.7\%). A study done by Iraj et al., (2013) showed that resistance to Ciprofloxacin was $63.3 \%$ and according to Upadhyay et al., resistance to cefepime was $64.7 \%$ which is also similar to our study (Upadhaya, 2014; Iraj et al., 2013). Present study shows least resistance against Imipenem (33.3\%), Piperacillin/Tazobactum (33.3\%), Colistin (16.7\%) similar study done by Iraj et al., obtained $23.3 \%$ resistance to Imipenem (Iraj et al., 2013) (Fig. 2-4). 
Fig.1 Prevalence of multidrug resistance Pseudomonas aeruginosa in samples studied

\section{Resistance pattern}

\section{$21 \%$}

Positive

$79 \%$
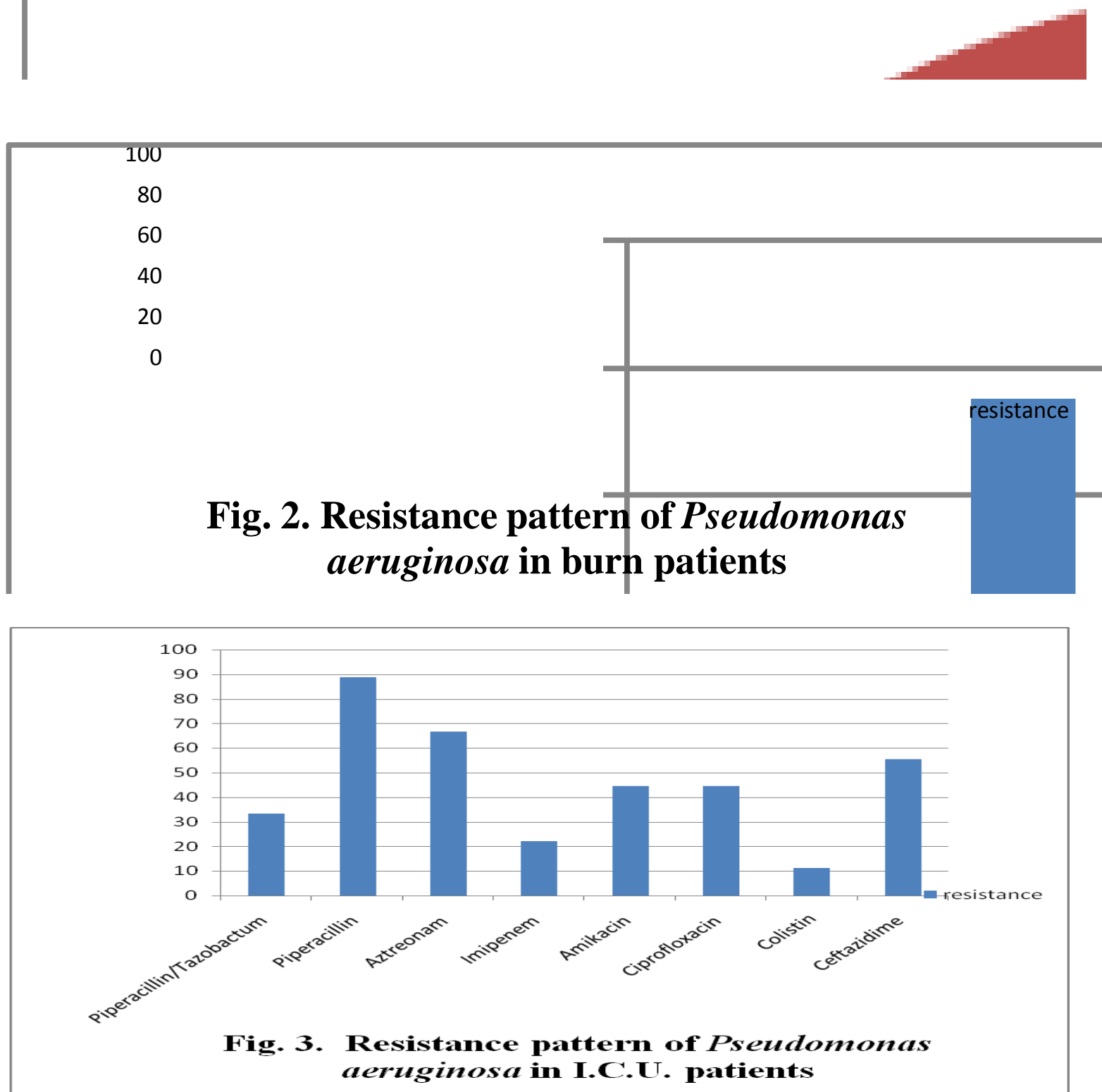
Fig.4 Multidrug resistance pattern of Pseudomonas aeruginosa

\section{Multidrug resistance \\ 0}

$100 \%$

Table.1 Resistance pattern of Pseudomonas aeruginosa in burn patients

\begin{tabular}{|c|}
\hline S. No. \\
\hline 1. \\
\hline 2. \\
\hline 3. \\
\hline 4. \\
\hline 5. \\
\hline 6. \\
\hline 7. \\
\hline 8. \\
\hline 9 \\
\hline
\end{tabular}

\begin{tabular}{|c|c|c|}
\hline Antibiotics & No. of samples $(n=12)$ & $\%$ \\
\hline Piperacillin $100 \mu \mathrm{g} /$ Tazobactum $10 \mu \mathrm{g}$ & 4 & 33.3 \\
\hline Piperacillin $100 \mu \mathrm{g}$ & 9 & 75 \\
\hline Aztreonam $30 \mu \mathrm{g}$ & 9 & 75 \\
\hline Imipenem $10 \mu \mathrm{g}$ & 4 & 33.3 \\
\hline Amikacin $30 \mu \mathrm{g}$ & 10 & 83.3 \\
\hline Ciprofloxacin $5 \mu \mathrm{g}$ & 7 & 58.3 \\
\hline Colistin10 $\mu \mathrm{g}$ & 2 & 16.7 \\
\hline Ceftazidime $30 \mu \mathrm{g}$ & 9 & 75 \\
\hline Cefepime & 8 & 66.7 \\
\hline
\end{tabular}

Table.2 Resistance pattern of Pseudomonas aeruginosa in I.C.U. patients

\begin{tabular}{|c|c|c|c|}
\hline S. No. & Antibiotics & $\begin{array}{r}\text { Resistance in No. of } \\
\text { samples }(\mathbf{n}=\mathbf{9})\end{array}$ & $\%$ \\
\hline 1. & Piperacillin $100 \mu \mathrm{g} /$ Tazobactum $10 \mu \mathrm{g}$ & $\mathbf{3}$ & $\mathbf{3 3 . 3}$ \\
\hline 2. & Piperacillin $100 \mu \mathrm{g}$ & $\mathbf{7}$ & $\mathbf{7 7 . 8}$ \\
\hline 3. & Aztreonam $30 \mu \mathrm{g}$ & $\mathbf{6}$ & $\mathbf{6 6 . 7}$ \\
\hline 4. & Imipenem $10 \mu \mathrm{g}$ & $\mathbf{2}$ & $\mathbf{2 2 . 2}$ \\
\hline 5. & Amikacin $30 \mu \mathrm{g}$ & $\mathbf{5}$ & $\mathbf{5 5 . 6}$ \\
\hline 6. & Ciprofloxacin $5 \mu \mathrm{g}$ & $\mathbf{4}$ & $\mathbf{4 4 . 4}$ \\
\hline 7. & Colistin $10 \mu \mathrm{g}$ & $\mathbf{1}$ & $\mathbf{1 1 . 1 1}$ \\
\hline 8. & Ceftazidime $30 \mu \mathrm{g}$ & $\mathbf{5}$ & $\mathbf{5 5 . 6}$ \\
\hline 9. & Cefepime & $\mathbf{4}$ & $\mathbf{4 4 . 4}$ \\
\hline
\end{tabular}


According to Kamaria et al., P. aeruginosa was $56.7 \%$ and $0 \%$ resistant to Piperacillin/Tazobactum and Colistin respectively (Kamaria et al., 2016).

ICUs are generally considered epicenters of antibiotic resistance and the principal sources of outbreaks of multi-resistant bacteria. The most important risk factors are obvious, such as excessive consumption of antibiotics exerting selective pressure on bacteria, the frequent use of invasive devices and relative density of a susceptible patient population with severe underlying diseases (Weber et al., 1999).

The prevalence of the $P$. aeruginosa isolates varied with the clinical conditions and the specimens. The highest number of isolates was from pus and endotracheal tubes.

Present study shows the highest resistance to piperacillin (77.8\%) and Aztreonam (66.7\%) similar study done by Lutfu savas et al., showed that Aztreonam was $63.1 \%$ resistant but Piperacillin was only $28.7 \%$ of resistant. (Lutfu savas et al., 2005) In our study P.aeruginosa shows intermediate resistance to Amikacin (55.6\%), Ceftazidime (55.6\%), Cefepime (44.4\%) and Ciprofloxacin (44.4\%) whereas Lutfu savas et al. showed that Amikacin, Ceftazidime, Cefepime and Ciprofloxacin were $42.2 \%, 48.9 \%, 39 \%$ and $27.4 \%$ resistant respectively (Lutfu savas et al., 2005).

Present study shows least resistance against Piperacillin/Tazobactum (33.3\%), Imipenem $(22.2 \%)$ and Colistin (11.11\%). Moreover, imipenem and combination therapy are effective antimicrobial agent used for treatment of $P$. aeruginosa similar study done by Masomeh Bayani et al., obtained 26.6\%, $40 \%$ resistance to Piperacillin/Tazobactum and Imipenem respectively (Masomeh Bayani et al., 2013) (Table 1 and 2).
However, possible explanations for the differences between our results with the findings of other studies are dissimilarity of antibiotics consumption, national and international antibiotics policy and hygiene measurement in different regions. In fact, the prevalence and resistance pattern of infectious agents are varied among the different hospitals in the same area or different regions throughout the world (Clark et al., 2003).

Pseudomonas aeruginosa has gradually become a major cause of nosocomial infections which occur in burn patients and which requires immediate and effective implementation of infection control strategies, to combat its spread. Environmental sources may play a significant role in spread of MDR among hospitalized patients. In current times, antibiotics with anti Pseudomonal activity which are available include the aminoglycosides, ticarcillin, penicillins and ciprofloxacin. Combination treatments are generally recommended for suspected Pseudomonas infections. It has been reported that the choice of cabapenem, piperacillin + tazobactum, ciprofloxacin and gentamicin combinations with amikacin in current times, appears to provide the widest potential antimicrobial activity against MDR P. aeruginosa (Ramprasad et al., 2010).

The lack of any new compounds in the near future indicates that national and local surveillance efforts are essential, to provide clinicians with correct information for choosing right antimicrobial therapy. Rigorous monitoring for MDR among Pseudomonas isolates is very important, because outbreaks caused by strains which are resistant to potentially useful agents, including carbapenems, have been reported elsewhere (Pfaller and Jones, 1997).

Thus, in ICUs, empirical antibiotic treatments should be avoided and treatment should be 
carried out using antibiotic susceptibility tests. ICUs should be regularly inspected for Pseudomonas colonization which shows a strong resistance pattern against the various antibiotics. Colonization of ICU patients with antimicrobial-resistant pathogens can lead to clinical infection because of breakdown of normal host defenses.

We conclude that Pseudomonas aeruginosa is highly resistant to Aminoglycosides, Fluoroquinolones and Penicillin group of drugs. Infection in burns continues to be a great problem which is not yet solved and poses a challenge to the microbiologists and the surgeons. Restriction of 'selected antibiotic usage' and/or infection control policies must be tailored for each institution, to combat the rapid emergence of MDR $P$. aeruginosa in burn patients. The lack of newer antimicrobial agents with activities against $P$. aeruginosa, makes periodic studies on the antimicrobial resistance patterns very important. We recommend regular screening of ICU patients to give an early warning of the presence of antimicrobial-resistant pathogens and allow the assessment of barrier and infection control techniques. Such monitoring can also aid infection control in determining how to focus efforts and reducing the emergence and spread of antimicrobial resistant pathogens.

\section{References}

Babay H.A.H., 2001-2005. Antimicrobial Resistance among Clinical Isolates of Pseudomonas aeruginosa from patients in a Teaching Hospital, Riyadh, Saudi Arabia, Jpn J Infect. Dis., 2007; 60:123125.

Appelgren $\mathrm{P}$, Bjornhagen $\mathrm{V}$, Bragderyd $\mathrm{K}$. 2002. A prospective study of infections in burn patients. Burns, 28: 39-46.

Steinsträsser L, Thies AH, Rabstein S, Steinau HU. 2007. Typical bacteria in an intensive care burn unit in severely burned patients and their importance with regard to mortality: retrospective study. Handchir Mikrochir Plast Chir., 39: 338-344.

Al-Tawfiq J A. 2007. Occurrence and antimicrobial resistance pattern of inpatient and outpatient isolates of Pseudomonas aeruginosa in a Saudi Arabian hospital: 1998-2003. Int J Inf Dis; 11:109-114.

Marilee D, Obritsch. 2005. Nosocomial infection due to Multidrug Resistant P.aeruginosa. Epidemiology and Treatment options. Pharmacotherapy, 25(10):1353-1364.

Carmeli, Y. N., Eliopoulos, G. M. and Samore, M. H. (2002). Antecedent Treatment with Different Antibiotic Agents. Emerg. Infect. Dis, 8: 802-807.

Koneman. 2006. The non-fermentative gramnegative Bacilli. In: Koneman s color atlas and textbook of diagnostic microbiology, Sixth edn, Williams and Wilkins, Lippincott. Pp. 303-391.

Clinical and Laboratory Standards Institute, Performance Standards for Antimicrobial Susceptibility Testing: Twentieth Informational Supplement M 100 S 20-U. Wayne, PA: CLSI, 2014.

Naseer S, Mabrouk A, Maher A. 2003. Colonization of Burn wounds in Ain Shams University Burn Unit. Burns, 29: 229-233.

Steinsträsser L, Thies AH, Rabstein S, Steinau HU. 2007. Typical bacteria in an intensive care burn unit in severely burned patients and their importance with regard to mortality: retrospective study. Handchir Mikrochir Plast Chir, 39: 338-344.

Upadhaya, S., 2014. Multi-drug Resistant Pseudomonas aeruginosa Isolated from Intensive Care Burn Unit, IJBR 05 (04).

Masomeh Bayani et al., 2013. Drug Resistance of Pseudomonas aeruginosa 
and Enterobacter cloacae Isolated from ICU, Babol, Northern Iran, Int J Mol Cell Med Autumn., 2(4): 205.

Lutfu savas et al., 2005. The Prevalence and Resistance Patterns of Pseudomonas aeruginosa in Intensive Care Units in a University Hospital, Turk J Med Sci., 35: 317-322.

Pooja A. Kamaria et al., July. 2016. Incidence of multidrug resistant Pseudomonas aeruginosa isolated from burn patients tertiary care hospital, Jamnagar, Gujarat, India. 15(7) Ver. VII, PP 3134.

Iraj N, Azita T, Zinab F, Kobra A, Saeedeh R, Mojtaba H, Sirous A, Afshin A. 2013. Antibiotic resistance and frequency of class 1 integrons among Pseudomonas aeruginosa, isolated from burn patients in Guilan, Iran. Iran J Microbiol., 5(1): 36-41.
Weber DJ, Raasch R, Rutala WA. 1999. Nosocomial infections in the ICU: the growing importance of antibioticresistant pathogens. Chest, 115: 34S$41 \mathrm{~S}$.

Clark NM, Patterson J, Lynch JP 2003. 3rd. Antimicrobial resistance among gramnegative organisms in the intensive care unit. Curr Opin Crit Care., 9: 413-23.

Ramprasad BP, Marissa R, Suprama D. 2010. Role of Pseudomonas in Nosocomial infections and Biological Characterization of Local Strains. $J$ Biosci Tech., 1(4): 170-9.

Pfaller MA, Jones RN, 1997. A review of the in vitro activity of meropenem and comparative antimicrobial agents tested against 30,254 aerobic and anaerobic pathogens isolated worldwide. Diagn Microbial Infect Dis., 28:157-63.

\section{How to cite this article:}

Usha Verma, Smita Kulshreshtha and Khatri, P.K.. 2018. MDR Pseudomonas aeruginosa in Nosocomial Infection: Burden in ICU and Burn Units of a Tertiary Care Hospital. Int.J.Curr.Microbiol.App.Sci. 7(01): 1267-1274. doi: https://doi.org/10.20546/ijcmas.2018.701.154 